\title{
ESTRATIFICAÇÃO DE RISCO PARA PÉ DIABÉTICO NUMA POPULAÇÃO DE IDOSOS ACOMPANHADOS NA ATENÇÃO PRIMÁRIA
}

\author{
RISK STRATIFICATION FOR DIABETIC FOOT IN A \\ POPULATION OF ELDERLY ATTENDED IN PRIMARY \\ HEALTH CARE
}

\section{ESTRATIFICACIÓN DEL RIESGO DE PIE DIABÉTICO EN UNA POBLACIÓN DE ANCIANOS ACOMPAÑADOS EN CUIDADOS PRIMARIOS}

\author{
Natália Pinheiro Fabrício Formiga ${ }^{1}$ \\ Paulo Renato Alves Firmino ${ }^{2}$ \\ Vitória de Cássia Félix Rebouças ${ }^{3}$ \\ Célida Juliana Oliveira ${ }^{4}$ \\ Márcio Flávio Moura de Araújo ${ }^{5}$ \\ Ana Maria Parente Garcia Alencar ${ }^{6}$
}

Como citar este artigo: Formiga NPF, Firmino PRA, Rebouças VCF, Oliveira CJ, Araújo MFM, Alencar AMPG. Estratificação de risco para pé diabético numa população de idosos acompanhados na atenção primária. Rev baiana enferm. 2020;34:e34097.

Objetivo: avaliar a estratificação de risco para pé diabético numa população de idosos acompanhados na atenção primária. Método: estudo transversal, analítico, com abordagem quantitativa. Realizaram-se visitas domiciliares a 254 idosos para avaliação neurológica (sensibilidade protetora plantar e sintomas neuropáticos), dermatológica e vascular (pulsos e índice tornozelo braquial). Resultados: parcela substancial (95,3\%) dos participantes referiu algum sintoma neuropático, sobretudo fadiga $(67,4 \%)$. A maioria apresentava risco para pé diabético $(64,1 \%)$, com predomínio do grau 1 (43,7\%); eram tabagistas (71,9\%), apresentavam comorbidade osteomuscular $(57,8 \%)$ e já tinham sofrido um AVC (75\%). As pessoas com grau de risco 2 e 3 tinham entre 10-19 desde o diagnóstico da doença (78,1\%). Conclusão: boa parte da amostra apresentava algum grau de risco para pé diabético, sobretudo do grau 1 , e presença de comorbidade osteomuscular.

Descritores: Idoso. Pé Diabético. Atenção Primária à Saúde.

Objective: to assess the risk stratification for diabetic foot in an elderly population followed up in primary care. Method: this is a cross-sectional, analytical study with a quantitative approach. Home visits were made to 254 elderly people for neurological (plantar protective sensitivity and neuropathic symptoms), dermatological and vascular (pulses and ankle brachial index) evaluation. Results: a substantial portion (95.3\%) of the participants reported some neuropathic symptom, especially fatigue (67.4\%). Most were at risk for diabetic foot (64.1\%), with a predominance of grade 1 (43.7\%); were smokers (71.9\%), had musculoskeletal comorbidity (57.8\%) and had already suffered a stroke

\footnotetext{
Enfermeira. Mestre em Enfermagem. Professora da Universidade Regional do Cariri. Crato, Ceará, Brasil. https://orcid.org/0000-0003-4589-9534

Estatístico. Doutor em Engenharia de Produção. Professor da Universidade Regional do Cariri. Crato, Ceará, Brasil. https://orcid.org/0000-0002-3308-2650

Enfermeira. Doutora em Enfermagem. Professora da Universidade Regional do Cariri. Crato, Ceará, Brasil. https://orcid.org/0000-0002-7890-7855.

Enfermeira. Doutora em Enfermagem. Professora da Universidade Regional do Cariri. Crato, Ceará, Brasil. https://orcid.org/0000-0002-8900-6833.

Enfermeiro. Doutor em Enfermagem. Professor da Fundação Oswaldo Cruz. Eusébio, Ceará, Brasil. marcio.moura@fiocruz.br. oicam29@gmail.com. https://orcid. org/0000-000 I-8832-8323.

6 Enfermeira e Obstetra. Doutora em Enfermagem. Professora da Universidade Regional do Cariri. Crato, Ceará, Brasil. https://orcid.org/0000-0003-0459-429।
} 
(75\%). People with risk levels 2 and 3 had had the diagnosis for between 10-19 years (78.1\%). Conclusion: a large part of the sample had some degree of risk for diabetic foot, in particular grade 1, and the presence of musculoskeletal comorbidity.

Descriptors: Elderly. Diabetic Foot. Primary Health Care.

Objetivo: evaluar la estratificación del riesgo para el pie diabético en una población anciana con seguimiento en atención primaria. Método: estudio analítico transversal con enfoque cuantitativo. Se realizaron visitas domiciliarias a 254 personas mayores para evaluación neurológica (sensibilidad protectora plantar y síntomas neuropáticos), evaluación dermatológica y vascular (índice de muñeca y tobillo braquial). Resultados: una porción sustancial (95,3\%) de los participantes informó algún síntoma neuropático, especialmente fatiga (67,4\%). La mayoría estaba en riesgo de pie diabético (64,1\%), con un predominio de grado 1 (43.7\%); eran fumadores (71,9\%), tenían comorbilidad musculoesquelética (57,8\%) y ya habian sufrido un derrame cerebral (75\%). Las personas con niveles de riesgo 2 y 3 tenían entre 10 y 19 años desde el diagnóstico de la enfermedad (78,1\%). Conclusión: una buena parte de la muestra tenía cierto grado de riesgo de pie diabético, especialmente de grado 1, y la presencia de comorbilidad musculoesquelética.

Descriptores: Ancianos. Pie Diabético Atención Primaria de Salud.

\section{Introdução}

O pé diabético é a presença de infecção, ulceração e/ou destruição de tecidos profundos que estão associados a anormalidades neurológicas e a vários graus de doença vascular periférica em pessoas com diabetes mellitus (DM). Trata-se de uma das principais complicações do DM e um fardo socioeconômico para pessoas acometidas e sistema público de saúde, especialmente nos países de baixa renda. As estimativas apontam que cerca de 15 a 25\% das pessoas com diabetes podem desenvolver úlceras no pé durante a vida. A prevalência desses casos está entre 1,3 a $12 \%{ }^{(1-2)}$.

No idoso é considerada uma das complicações mais devastadoras, em função do grande número de casos que evoluem para amputação, com grande impacto socioeconômico, incluindo gastos com tratamentos, além de internações prolongadas e recorrentes ${ }^{(3)}$. Muitos fatores estão envolvidos no desenvolvimento dessa complicação. Ressalta-se que a maioria das amputações de pé diabético é precedida de ulcerações.

Nessa gênese, destacam-se a Polineuropatia Diabética e a Doença Arterial Periférica (DAP) que, associadas à mobilidade articular limitada e ao trauma repetitivo, propiciam a formação de úlceras e contribuem para a amputação de membros inferiores ${ }^{(4)}$. Atreladas a esses fatores, as alterações específicas do idoso, como envelhecimento cerebral, problemas osteoarticulares e a presença de catarata associada a retinopatia diabética, podem impactar de forma negativa nas atividades de autocuidado e somatizar a ocorrência do pé diabético nessa população ${ }^{(5)}$.

Todavia, evidências apontam que a ulceração de pé diabético é prevenível e o primeiro passo na prevenção de amputações é a cuidadosa triagem para identificação dos problemas nos pés e a detecção de pacientes de alto risco, o que aponta para a necessidade do desenvolvimento de estudos com foco nessa temática ${ }^{(2)}$.

Diretrizes internacionais e nacionais recomendam a avaliação clínica, neurológica e vascular dos pés das pessoas com diabetes, em todas as suas etapas, como prioridade no planejamento do cuidado. Os resultados obtidos na avaliação poderão nortear o planejamento de ações em saúde contextualizadas e efetivas na prevenção do pé diabético ${ }^{(4-6)}$.

Nessa direção, um dos principais grupos de especialistas globais nessa temática recomendam que todos os pacientes com DM tipo 2 (DM2) devem ser examinados anualmente para a presença de DAP, incluindo história detalhada, palpação dos pulsos e cálculo do Índice Tornozelo Braquial (ITB), além de receberem orientações para a cessação do tabagismo e de outros fatores de risco identificados ${ }^{(1)}$. Contudo, no Brasil, 
autores argumentam que poucas pesquisas realizam o cálculo do ITB. Desse modo, a estratificação do risco para pé diabético é mensurada apenas com base na palpação dos pulsos e de sinais clínicos de DAP ${ }^{(7)}$.

O objetivo deste estudo é avaliar a estratificação de risco para pé diabético numa população de idosos acompanhados na atenção primária.

\section{Método}

Trata-se de estudo transversal, analítico, com abordagem quantitativa. A coleta de dados ocorreu no período de julho de 2016 a janeiro de 2017 em unidades de atenção primária à saúde da zona urbana do município de Juazeiro do Norte, Ceará, Brasil. O estudo foi aprovado pelo Comitê de Ética em Pesquisa com Seres Humanos da Universidade Regional do Cariri sob Parecer n. 1.536.396. Todos os participantes manifestaram sua anuência mediante a assinatura do Termo de Consentimento Livre e Esclarecido.

$\mathrm{Na}$ ocasião do estudo, o quantitativo de idosos com DM2 e em serviços de atenção primária da zona urbana era de aproximadamente de 2.500 pessoas. Com base nestas informações, obteve-se o dimensionamento da amostra por meio da fórmula para população finita, tomando como parâmetros a prevalência conservadora de 50\% e erro amostral de 5\%, resultando em 254 idosos com DM2. Utilizou-se a técnica de amostragem estratificada proporcional para seleção do quantitativo de idosos por serviço de saúde selecionado para o estudo. Foram visitadas 67 equipes de ESF do município que realizavam acompanhamento das pessoas com diabetes, no que diz respeito aos planos alimentar, medicamentoso e exercício físico. Contudo, em apenas 49 indivíduos, foi possível coletar dados, devido às questões organizacionais, como unidades básicas de saúde em greve, por falta de recursos humanos e/ou materiais para o funcionamento do serviço para a população, o que dificultou a captação dos idosos.

Os participantes foram selecionados por conveniência, isto é, foram abordados nos serviços nos dias de consulta agendados para pessoas com diabetes. Na sequência, foram programadas visitas domiciliares para captação de dados relacionados ao exame físico dos idosos. Elencamos como critérios de inclusão no estudo: ter idade $\geq 60$ anos, diagnóstico de DM2 e ser cadastrado numa ESF do município. Dentre os elegíveis, 44 participantes foram excluídos devido aos seguintes critérios de exclusão: não compareceram ao serviço ou não estavam em seus domicílios durante a coleta de dados (23); recusaram-se a integrar o estudo (5); óbito (1); negaram ter DM2 (6); endereço não encontrado (5); falta de registros clínicos (2); comportamento ou comunicação alterada, que comprometesse sua participação na entrevista (1); diagnóstico concomitante de hanseníase (1).

No domicílio dos participantes, foi realizada a entrevista e o exame físico em ambiente privativo, mediante a aplicação de dois instrumentos. No primeiro foram contempladas variáveis relacionadas à caracterização sociodemográfica (sexo, idade, estado civil, escolaridade, renda e arranjo familiar) e clínica (tempo de diagnóstico da doença, tabagismo, etilismo, prática de atividade física, comorbidades e complicações); no segundo instrumento. exploraram-se variáveis relacionadas à situação dermatológica, vascular e neurológica dos pés dos idosos com DM2, por meio de avaliação e rastreamento da dor neuropática, perda da sensibilidade protetora (PSP) e DAP. Isto, conforme as diretrizes da Sociedade Brasileira de Diabetes (SBD) e do Grupo Internacional do Pé Diabético ${ }^{(1,6)}$.

Para o rastreamento da dor neuropática, foram consideradas as características dos sintomas neuropáticos - queimação, dormência, formigamento, câimbras e dor - cuja intensidade foi avaliada pela escala Wong-Baker de Faces de Dor ${ }^{(1)}$.

$\mathrm{Na}$ avaliação dermatológica, consideraram-se as variáveis presença ou não de vasos dilatados dorsais, pele seca, rachaduras, fissuras, cor da pele normal, micose interdigital, micose ungueal, pelos presentes, calosidades, calçados adequados, avaliadas mediante inspeção dos pés. Sobre a avaliação musculoesquelética, verificou-se os pés quanto à presença das deformidades do tipo 
acentuação ou queda do arco plantar, joanetes e dedos em garra.

Durante a verificação neurológica dos pés, para avaliação da perda da sensibilidade protetora plantar (PSP), utilizaram-se o monofilamento Semmes-Weinstein de $10 \mathrm{~g}$ associado ao diapasão de $128 \mathrm{~Hz}$, como recomendado para diagnóstico da PSP $^{(1,6)}$.

Aplicou-se o monofilamento referido com o idoso deitado e em quatro áreas plantares: hálux (falange distal), primeiro, terceiro e quinto metatarsos, por três tentativas em cada local, duas positivas e outra simulada. Já o teste do diapasão de $128 \mathrm{~Hz}$ foi aplicado na falange distal do hálux, de forma perpendicular com pressão constante, por três tentativas no local, duas positivas e outra simulada da vibração do aparelho. Em casos de amputações menores, impossibilitando-se a verificação, foi aplicado na região do maléolo, conforme recomendação da literatura, por se tratar da proeminência óssea mais próxima ${ }^{(1,6)}$.

Quanto à avaliação vascular, realizou-se a palpação dos pulsos dorsais e tibiais posteriores em ambos os pés. A triagem de DAP ocorreu por meio do cálculo de ITB, identificação de amputação e úlceras prévias e ativas. A técnica de verificação do ITB consistiu na aferição da pressão arterial braquial sistólica, utilizando-se esfigmomanômetro e estetoscópio, conforme técnica recomendada pelas Diretrizes da Sociedade Brasileira de Hipertensão ${ }^{(8)}$. Em seguida, aferiu-se a pressão arterial sistólica das artérias distais de ambos os MMII, por meio do esfigmomanômetro, com manguito acima dos pulsos (artéria tibial posterior e artéria pediosa dorsal), utilizando-se o doppler vascular manual de transdutor de 8 a 10 MHz marca MEDPEJ ${ }^{\circledR}$ modelo DF-70001 VN, sobre o pulso arterial ${ }^{(6)}$.

Foram obtidos também os dados antropométricos com aferição de peso e altura para cálculo do Índice de Massa Corporal (IMC), utilizando-se os pontos de corte menor ou igual a $22 \mathrm{~kg} / \mathrm{m}^{2}$ (baixo peso), maior que 22 e menor que $27 \mathrm{~kg} / \mathrm{m}^{2}$ (eutrofia), maior ou igual $27 \mathrm{~kg} / \mathrm{m}^{2}$ (sobrepeso), recomendados para o idoso ${ }^{(9)}$.

Após cálculo dos valores, dividiu-se o maior valor obtido das artérias distais de ambos os MMII pelo maior valor das artérias braquiais aferido bilateralmente. Os pontos de corte foram: ITB $<0,9$ (DAP), 0,9 a 1,30 (normal), ITB >1,30 (Calcificação arterial) $^{(1)}$. Como resultado da avaliação, foi estratificado o risco de desenvolvimento de pé diabético de acordo com a SBD, utilizando-se os escores de risco 0 (PSP e DAP ausentes), 1 (PSP associada ou não a deformidade), 2 (DAP associada ou não a PSP) e 3 (histórico de ulceração ou amputação) $)^{(6)}$.

Analisaram-se os dados mediante a estatística descritiva e a inferencial com testes de hipóteses e modelagem, por meio do Software Estatístico R na versão RStudio 1.0.136. Para a avaliação de correlação de dependência entre as variáveis, foram utilizados os testes Teste Qui-quadrado de Pearson e Teste de correlação de Spearman. Para a análise da sensibilidade da variação do nível de significância $(\alpha)$ e o resultado dos testes (p-value), adotou-se o nível de 5\% $(\alpha<0,05)$.

\section{Resultados}

A amostra foi composta predominantemente por mulheres $(71,7 \%)$ com até oito anos de estudo (50,4\%), casadas (42,5\%) e aposentadas (70,9\%). Quanto à idade, os idosos participantes tinham em média $73,3 \pm 7,8$ anos na ocasião da pesquisa.

Os participantes conviviam com o diabetes em média a 10,1 \pm 8 anos e tinham como terapêutica majoritária a monoterapia com antidiabético oral (76\%). A maioria não fazia uso do tabaco $(87,4 \%)$ ou álcool $(92,1 \%)$ e eram sedentários (80,3\%). Boa parte $(42,9 \%)$ também estava acima do peso, especialmente as mulheres (45,1\%). Dentre os sobrepesados predominou a obesidade tipo 1 (44\%).

Identificou-se que 90,6\% dos participantes apresentaram alguma comorbidade, com prevalência de hipertensão arterial (89,7\%). Acerca disso, pode-se também esclarecer que as mulheres eram mais acometidas por comorbidades, com 50\% delas apresentando de 1-3 comorbidades $(\mathrm{p}=0,002)$. Boa parte da amostra (76\%) já apresentava alguma complicação do diabetes, com predomínio das oftalmológicas (88,1\%), como, por exemplo, diminuição progressiva da acuidade visual $(61,2 \%)$ e, inclusive, cegueira $(8,2 \%)$. 
Outro fato é que $23,9 \%$ já tinham sofrido um infarto. Destaca-se que as pessoas sem complicação decorrente do diabetes apresentaram tempo de diagnóstico menor que 20 anos $(\mathrm{p}<0.000)$.

Parcela substancial $(95,3 \%)$ dos participantes referiu algum sintoma neuropático, destacando-se fadiga $(67,4 \%)$, dor $(62,4 \%)$ e câimbras
(61,2\%). No quesito dor, vale destacar que em $33,9 \%$ a intensidade era "moderada". Na avaliação dermatológica, identificaram-se pele seca, rachaduras e fissuras em quase todos os participantes (96,5\%). Ressalta-se que todos os indivíduos apresentaram mais de uma das características apresentadas na Gráfico 1.

Gráfico 1 - Distribuição das características dos pés dos participantes, conforme avaliação dermatológica. Juazeiro do Norte, Ceará, Brasil - 2017

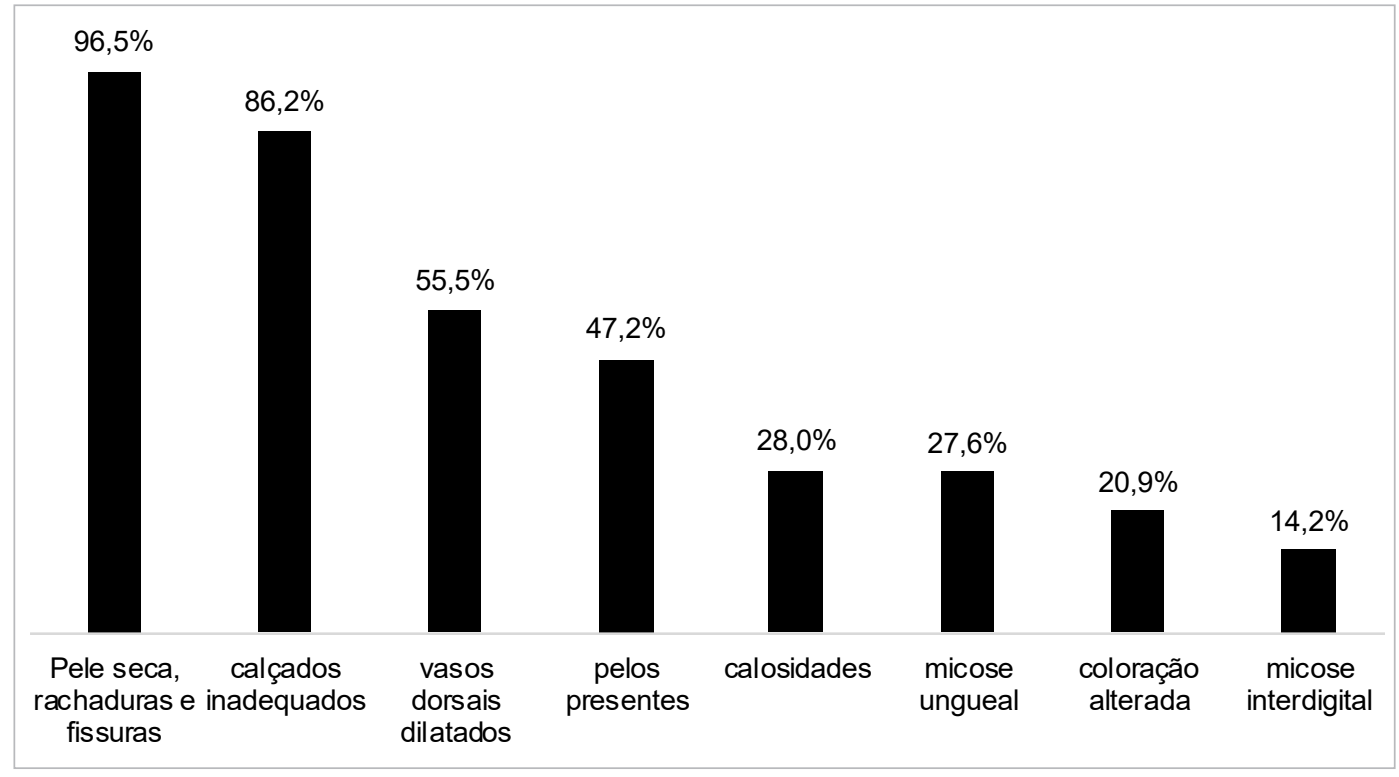

Fonte: Elaboração própria.

Independente do sexo, o problema dermatológico mais constatado no exame dermatológico foi a pele seca, com rachaduras e fissuras. Observou-se maior disparidade entre os sexos nos itens calçados inadequados e micose ungueal, com maior acometimento do sexo feminino e masculino, respectivamente (Gráfico 2).

Gráfico 2 - Distribuição das características da avaliação dermatológica dos pés dos participantes, conforme o sexo. Juazeiro do Norte, Ceará, Brasil - 2017

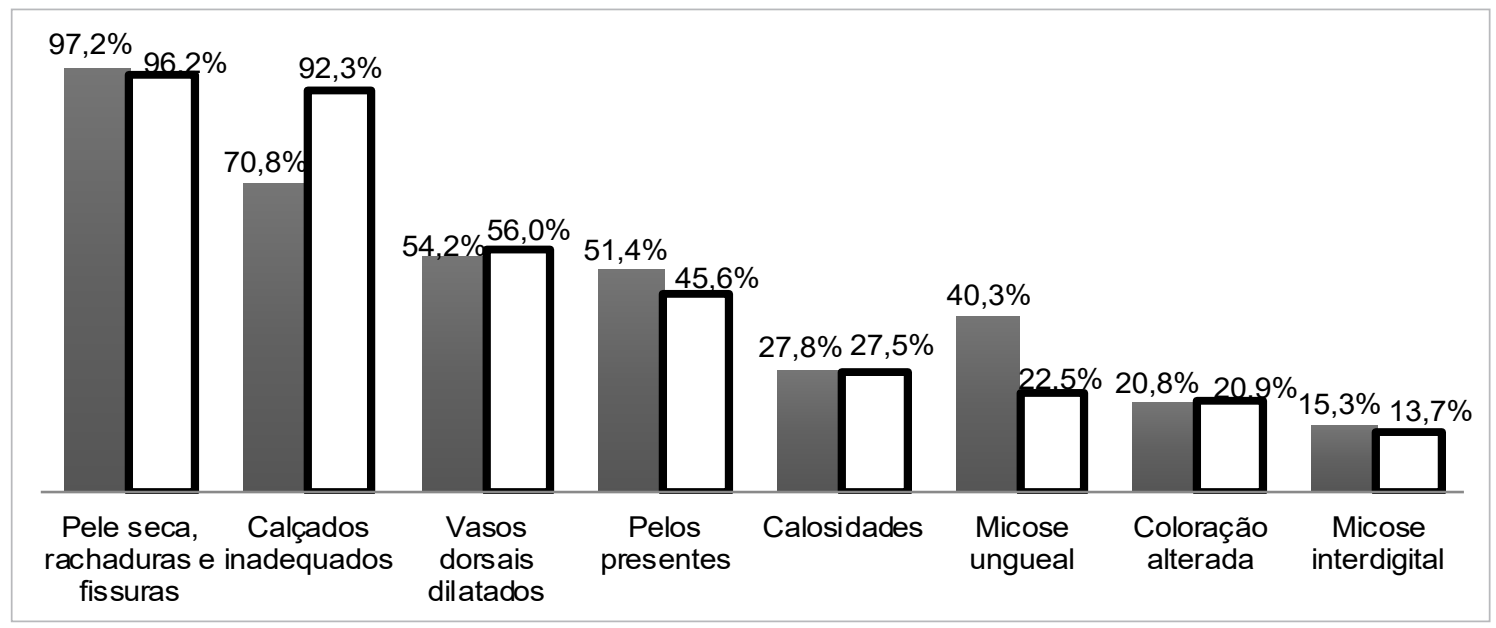

Legenda: - Masculino $\square$ Feminino 
Quanto à avaliação vascular, a maioria dos participantes apresentou pulsos (78,3\%) e classificação de ITB (53,5\%). Identificou-se, no estudo, prevalência de ITB anormal, sugestivo de DAP, de 7,1\%, sendo o sexo feminino $(8,2 \%)$ acometido praticamente duas vezes mais que o masculino (4,2\%).

Apesar de apenas 5,5\% apresentarem amputação prévia, 15\% já apresentavam úlcera e 6,3\% úlcera ativa, com predomínio das ulcerações do tipo neuropáticas $(68,8 \%)$, em seguida neuroisquêmicas $(18,8 \%)$ e isquêmicas $(6,3 \%)$.

Com base nesses dados, foi constatado que $43,7 \%$ dos participantes apresentavam risco para pé diabético grau 1, seguido daqueles com grau $0(35,8 \%)$, grau $3(15,7 \%)$ e grau $2(4,7 \%)$.
Entre os idosos com algum grau de risco para pé diabético, os percentuais foram maiores no estrato de maior idade ( $\geq 80$ anos) $(p=0,002)$. Em todas as faixas de renda, aproximadamente metade dos participantes (40,5\%, 52,5\% e 50\%) foi classificado com risco para pé diabético Grau 1 $(\mathrm{p}=0,024)$. As pessoas sem nenhum grau de risco, assim como aquelas com Grau 2 e 3 para pé diabético. tinham menos de 10 anos de doença (somatório dos menores estratos apresentados) ( $\mathrm{p}=0,003)$. Aproximadamente $40 \%$ das pessoas com grau 1 de risco para pé diabético eram tabagistas $(p=0,018)$, apresentavam comorbidade osteomuscular $(\mathrm{p}=0,021)$ e já tinham sofrido um AVC ( $\mathrm{p}=0,018)$ (Tabela 1$)$.

Tabela 1 - Distribuição numérica e percentual da classificação de risco de desenvolvimento de pé diabético, conforme variáveis sociodemográficas e clínicas dos idosos com diabetes tipo 2. Juazeiro do Norte, Ceará, Brasil - 2017 (n=91)

(continua)

\begin{tabular}{|c|c|c|c|c|c|c|c|c|c|}
\hline \multirow{3}{*}{ Variáveis } & \multicolumn{8}{|c|}{ Classificação de risco para pé diabético } & \multirow{3}{*}{ p-valor } \\
\hline & \multicolumn{2}{|c|}{ Grau 0} & \multicolumn{2}{|c|}{ Grau 1} & \multicolumn{2}{|c|}{ Grau 2} & \multicolumn{2}{|c|}{ Grau 3} & \\
\hline & $\mathbf{n}$ & $\%$ & $\mathbf{n}$ & $\%$ & $\mathbf{n}$ & $\%$ & $\mathbf{n}$ & $\%$ & \\
\hline Sexo & & & & & & & & & 0,307 \\
\hline Feminino & 63 & 34,6 & 84 & 46,2 & 10 & 5,5 & 25 & 13,7 & \\
\hline Masculino & 28 & 38,9 & 27 & 37,5 & 2 & 2,8 & 15 & 20,8 & \\
\hline \multicolumn{10}{|l|}{ Idade } \\
\hline $60-69$ anos & 48 & 45,7 & 38 & 36,2 & 3 & 2,9 & 16 & 15,2 & $0,002 *$ \\
\hline $70-79$ anos & 32 & 32,3 & 47 & 47,5 & 6 & 6,1 & 14 & 14,1 & \\
\hline De 80 anos acima & 11 & 22,0 & 26 & 52,0 & 3 & 6,0 & 10 & 20,0 & \\
\hline \multicolumn{10}{|l|}{ Renda familiar } \\
\hline 1 - 2 salários mínimos & 68 & 36,8 & 75 & 40,5 & 9 & 4,9 & 33 & 17,8 & $0,024^{*}$ \\
\hline 3 - 4 salários mínimos & 12 & 30,0 & 21 & 52,5 & 1 & 2,5 & 6 & 15,0 & \\
\hline Mais de 5 salários mínimos & 1 & 25,0 & 2 & 50,0 & - & - & 1 & 25,0 & \\
\hline \multicolumn{10}{|l|}{ Tempo de diabetes } \\
\hline$<10$ anos & 51 & 72,9 & 1 & 1,4 & 6 & 8,6 & 12 & 17,1 & \\
\hline $10-19$ anos & 26 & 21,8 & 62 & 52,1 & 5 & 4,2 & 26 & 21,8 & $0,003^{*}$ \\
\hline $20-29$ anos & 12 & 24,5 & 36 & 73,5 & 1 & 2,0 & - & - & \\
\hline $30-39$ anos & 2 & 2,9 & 10 & 14,3 & - & - & 2 & 2,9 & \\
\hline De 40 anos acima & - & - & 3 & 100 & - & - & - & - & \\
\hline Tabagismo & 9 & 28,1 & 14 & 43,8 & 5 & 15,6 & 4 & 12,5 & $0,018^{*}$ \\
\hline Etilismo & 7 & 35,0 & 9 & 45,0 & - & - & 4 & 20,0 & 0,732 \\
\hline \multicolumn{10}{|l|}{ Comorbidades } \\
\hline HAS & 72 & 34,3 & 93 & 44,3 & 12 & 5,7 & 33 & 15,7 & 0,233 \\
\hline Dislipidemias & 45 & 37,2 & 49 & 40,5 & 8 & 6,6 & 19 & 15,7 & 0,494 \\
\hline Osteomuscular & 34 & 28,1 & 54 & 44,6 & 7 & 5,8 & 9 & 7,4 & $0,021^{*}$ \\
\hline \multicolumn{10}{|l|}{ Complicações } \\
\hline Renal & 7 & 26,9 & 12 & 46,2 & 1 & 3,8 & 6 & 23,1 & 0,822 \\
\hline Oftalmológicas & 52 & 30,6 & 80 & 47,1 & 10 & 5,9 & 28 & 16,5 & 0,907 \\
\hline Cardiovascular & 12 & 26,1 & 24 & 52,2 & 1 & 2,2 & 9 & 19,6 & 0,537 \\
\hline $\mathrm{AVC}$ & 9 & 25,0 & 16 & 44,4 & 6 & 16,7 & 5 & 13,9 & $0,018^{*}$ \\
\hline
\end{tabular}


Tabela 1 - Distribuição numérica e percentual da classificação de risco de desenvolvimento de pé diabético, conforme variáveis sociodemográficas e clínicas dos idosos com diabetes tipo 2. Juazeiro do Norte, Ceará, Brasil - 2017 ( $\mathrm{n}=91)$ (conclusão)

\begin{tabular}{|c|c|c|c|c|c|c|c|c|c|}
\hline \multirow{3}{*}{ Variáveis } & \multicolumn{8}{|c|}{ Classificação de risco para pé diabético } & \multirow{3}{*}{ p-valor } \\
\hline & \multicolumn{2}{|c|}{ Grau 0} & \multicolumn{2}{|c|}{ Grau 1} & \multicolumn{2}{|c|}{ Grau 2} & \multicolumn{2}{|c|}{ Grau 3} & \\
\hline & $\mathbf{n}$ & $\%$ & $\mathbf{n}$ & $\%$ & $\mathbf{n}$ & $\%$ & $\mathbf{n}$ & $\%$ & \\
\hline \multicolumn{10}{|c|}{ Prática de atividade física } \\
\hline $\operatorname{Sim}$ & 25 & 50,0 & 20 & 40,0 & 1 & 2,0 & 4 & 8,0 & 0,070 \\
\hline Não & 66 & 32,4 & 91 & 44,6 & 11 & 5,4 & 36 & 17,6 & \\
\hline
\end{tabular}

Fonte: Elaboração própria.

Notas: Sinal convencional utilizado:

- Dado numérico igual a zero não resultante de arredondamento.

* $p<0,05$ = significância estatística.

\section{Discussão}

Essencialmente, o estudo foi composto por mulheres idosas com mais de 10 anos de DM2 e com alguma complicação da doença já instalada. Estudos prévios apontaram dados similares acerca deste perfil ${ }^{(10-11)}$. Estilo de vida e estado geral de saúde das mulheres brasileiras têm elevado o surgimento de casos de DM2, além da questão de maior sobrevida em relação aos homens. A propósito, dados já mostram, no Brasil, essa tendência nos últimos dez anos. O percentual de mulheres com diabetes, em 2018, passou de $6,7 \%$ para $8,1 \%$, contra um crescimento de $5,7 \%$ para $7,1 \%$ entre os homens ${ }^{(12)}$.

A queixa neuropática predominante nesta pesquisa foi a fadiga. Fato divergente de estudo prévio de delineamento similar que evidenciou maior prevalência para queimação, dormência ou formigamento ${ }^{(7)}$. Todavia, é importante salientar que esse estudo investigou não somente pessoas idosas, mas também pessoas com idade a partir de 10 anos.

O autorrelato de fadiga favorece a identificação precoce de problemas mais severos, como a dor isquêmica provocada por doença vascular periférica. Desse modo, além do reconhecimento do sintoma, é necessário avaliar a fadiga e a dor associadas quanto à sua intensidade, qualidade, localização, duração e impacto no idoso com diabetes como rotina por ocasião do atendimento em saúde ${ }^{(5)}$.
Um possível fator agravante neste caso seria o sedentarismo desta amostra, que foi considerável, já que a prática de atividade física regular é favorável à perfusão tissular e à prevenção das complicações micro e macro vasculares do DM.

Acerca da avaliação dermatológica, foi substancial o relato de alterações como pele seca, rachaduras e fissuras nos pés dos participantes. Pesquisas conduzidas com um público de pessoas com DM em serviços de atenção primária e em ambulatórios especializados obtiveram o mesmo achado ${ }^{(7,13-15)}$.

Pessoas com DM já possuem derme e epiderme menos espessa e com menor hidratação cutânea. Por isso, existe a necessidade de cuidados adicionais relativos à orientação sobre a prevenção de ressecamento da pele com agentes emolientes e prevenção de micoses com o uso de antifúngicos e secagem dos espaços interdigitais após a lavagem dos pés, por meio de sessões de educação em saúde nos diversos cenários da rede de atenção à saúde no país, respeitando-se possíveis diferenças regionais num país continental como o Brasil.

A predominância de rachaduras, fissuras e pele seca em homens e mulheres não causou estranheza. Como exemplo, cita-se a predominância de uso de calçados errados entre as mulheres, por ser um acessório com mais opções para esse público e, culturalmente, item preferível ao público feminino no cotidiano. 
Tal achado também foi verificado em outra publicação, que identificou, entre as mulheres, maior prevalência de cuidados necessários para prevenir lesões e, nos homens, melhores hábitos relacionados ao calçado adequado ${ }^{(16)}$. Entretanto, acerca disso, é possível que justamente a vaidade feminina no uso dos calçados possa ter interferido no percentual apresentado nesta pesquisa.

Importante destacar que o uso de calçados inadequados pelos idosos deste estudo pode favorecer o aparecimento de lesões intimamente relacionadas ao desenvolvimento de deformidades, cisalhamento do calçado e infecção, que predispõem ao desenvolvimento do pé diabético e maiores complicações ${ }^{(17)}$.

Neste sentido, a seleção de calçados é considerada uma prescrição e deve envolver critérios clínicos e confecção dentro de normas padronizadas pelos consensos e diretrizes ${ }^{(4,6)}$. Contudo, os calçados adequados para pessoas com diabetes apresentam custo elevado, dificultando o acesso pela maioria dos pacientes ${ }^{(18)}$. Tendo em vista o baixo nível econômico dos idosos deste estudo, outra explicação seria a realidade social e econômica dos integrantes da amostra.

Neste estudo, a classificação de risco de desenvolvimento de pé diabético predominante foi de risco grau 1. Os dados da literatura são divergentes neste ponto, contudo a maioria dos estudos consultados identificou prevalência maior para risco grau 1 em relação a este estudo ${ }^{(15,17,19)}$.

Averiguou-se ainda um quantitativo considerável de publicações nas quais predominaram pessoas com risco 0 para pé diabético. Entretanto, destaca-se que boa parte não realizou cálculo ITB, mas apenas a palpação dos pulsos associada aos sinais clínicos de DAP para inferir e classificar as pessoas avaliadas ${ }^{(14,20-22)}$.

Observou-se, nesta investigação, que as pessoas com risco grau 1 apresentaram associação com variáveis como tabagismo, renda, comorbidade osteomuscular, AVC e idade avançada ( $>80$ anos).

O tabaco é um importante fator de risco cardiovascular, desencadeador de alterações endoteliais que comprometem a cicatrização e aumentam o risco de ulceração nos pés. É sabido também que o seu uso causa estresse oxidativo celular, preditor de neuropatia diabética e resistência insulínica ${ }^{(23)}$. Ademais, o tabaco aumenta as taxas de DAP, diminui a capacidade de transporte de oxigênio, resultando em hipóxia tecidual, prejudicando a cicatrização das lesões e aumentando o risco das amputações ${ }^{(24)}$. Portanto, o enfrentamento do tabagismo é crucial na prevenção do pé diabético.

A associação averiguada com AVC decorre possivelmente da mesma causa do uso do tabaco, pois o histórico desse evento já indica a presença prévia de vascularização prejudicada nesses pacientes. Ademais, a presença de DAP é um marcador de aterosclerose nos leitos vasculares cardíacos e cerebrais $^{(25)}$. Infere-se também que as pessoas com diabetes e sequelas de AVC podem apresentar comprometimento neurológico que comprometa a manutenção de um estilo de vida ativo e nas práticas de autocuidado com os pés.

A prática do autocuidado pode também estar prejudicada em pessoas com complicações osteomusculares em virtude do comprometimento da carga biomecânica do pé, gerando pontos de pressão anormais, que podem predispor às deformidades e neuropatias diabéticas ${ }^{(1)}$.

Uma evidência estatisticamente significante observada neste estudo foi o predomínio de casos de grau de risco 1 naqueles com maior estrato de idade investigado (>80 anos). Por outro lado, constatou-se também, com relevância estatística, que os casos de grau 2 e 3 predominaram naqueles cujo tempo de doença era $<20$ anos. Isto leva a refletir-se que o avançar da idade e suas particularidades vascular, dermatológica e neurológica podem favorecer o menor risco de desenvolvimento de pé diabético (grau 1). Todavia, os casos de maior vulnerabilidade para pé diabético (grau 2 e 3 ) ocorrem em quem convive há menos de 20 anos com o DM, possivelmente mais jovem, mas com um controle metabólico da doença e autocuidado dos pés deficitários.

Este estudo apresenta como limitação a não avaliação de causa e efeito dos achados.

\section{Conclusão}

A maioria dos participantes da amostra sob estudo apresentava algum grau de risco para pé diabético $(64,1 \%)$, com predomínio do grau de 
risco $1(43,7 \%)$. Identificou-se que idosos com DM2 e risco grau 1 para pé diabético são mais prevalentes entre os tabagistas, aqueles com idade avançada (>80 anos), histórico de AVC e presença de comorbidade osteomuscular.

Esta pesquisa sinaliza a necessidade de mais estudos que possam ampliar a investigação para a população rural, outras faixas etárias e incluir, além do controle glicêmico capilar, a hemoglobina glicada. Contudo, as evidências apontaram subsídios para construção de estratégias educativas e prevenção do pé diabético em diferentes graus, otimizando o cuidado integral da pessoa com diabetes.

\section{Colaborações:}

1 - concepção, projeto, análise e interpretação dos dados: Natália Pinheiro Fabrício Formiga, Paulo Renato Alves Firmino e Ana Maria Parente Garcia Alencar;

2 - redação do artigo e revisão crítica relevante do conteúdo intelectual: Natália Pinheiro Fabrício Formiga, Márcio Flávio Moura de Araújo e Ana Maria Parente Garcia Alencar;

3 - aprovação final da versão a ser publicada: Natália Pinheiro Fabrício Formiga, Vitória de Cássia Félix Rebouças, Célida Juliana Oliveira e Ana Maria Parente Garcia Alencar.

\section{Referências}

1. International Working Group on Diabetic Foot. Guidelines on the prevention and management of diabetic foot disease. Maastricht; 2019 [cited 2019 Sep 20]. Available from: https://iwgdfguidelines. org/wp-content/uploads/2019/05/IWGDFGuidelines-2019.pdf

2. Yazdanpanah L, Shahbazian H, Nazari I, Arti HR, Ahmadi F, Mohammadianinejad SE, et al. Incidence and Risk Factors of Diabetic Foot Ulcer: A Population-Based Diabetic Foot Cohort (ADFC Study)-Two-Year Follow-Up Study. Int J Endocrinol. 2018 Mar;2018:7631659. DOI: $10.1155 / 2018 / 7631659$

3. Almeida AS, Silveira MM, Espírito Santo PF, Pereira RC, Salomé GM. Avaliação da qualidade de vida em pacientes com diabetes mellitus e pé ulcerado. Rev Bras Cir Plást.
2013;28(1):142-6. DOI: http://dx.doi.org/10.1590/ S1983-51752013000100024

4. Bakker K, Schaper NC, International Working Group on Diabetic Foot Editorial Board. The development of global consensus guidelines on the management and prevention of the diabetic foot 2011. Diabetes Metab Res Rev. 2012 Feb;28(suppl 1):S116-8. DOI: 10.1002/dmrr.2254

5. American Diabetes Association. Microvascular complications and foot care; Standards of Medical Care in Diabetes-2019. Diabetes care. 2019;42(Suppl 1):S124-38. DOI: 10.2337/dc19-S011

6. Sociedade Brasileira de Diabetes. Diretrizes da Sociedade Brasileira de Diabetes. 2019-2020. São Paulo: Clannad Editora Científica; 2019.

7. Braga DC, Bortolini SM, Rozetti IG, Zarpellon K, Nascimento JC, Neris JE. Avaliação de neuropatia e complicações vasculares em pacientes com diabetes mellitus em um município rural de Santa Catarina. Rev AMRIGS [Internet]. 2015 abr-jun [cited 2019 Aug 20];59(2):78-83. Available from: https://pesquisa.bvsalud.org/portal/resource/pt/ biblio-833930

8. Sociedade Brasileira de Cardiologia, Sociedade Brasileira de Hipertensão, Sociedade Brasileira de Nefrologia. 7aㅡ Diretriz Brasileira de Hipertensão. Arq Bras Cardiol [Internet]. 2016 [cited 2019 Aug 13];107(3):1-83. Available from: http:// publicacoes.cardiol.br/2014/diretrizes/2016/05_ HIPERTENSAO_ARTERIAL.pdf

9. Lipschitz DA. Screening for nutritional status in the elderly. Prim Care [Internet]. 1994 Mar [cited 2019 Sep 28];21(1):55-67. Available from: https://www. ncbi.nlm.nih.gov/pubmed/8197257

10. Martin IS, Beraldo AA, Passeri SM, Freitas MCF, Pace AE. Causas referidas para o desenvolvimento de úlceras em pés de pessoas com diabetes mellitus. Acta paul enferm. 2012;25(2):218-24. DOI: http:// dx.doi.org/10.1590/S0103-21002012000200010

11. Przysiezny A, Rodrigues KF, Santiago LH, Silva MCV. Características sociodemográficas de pacientes com diabetes mellitus portadores de pé diabético e ou retinopatia diabética atendidos em 16 unidades de Estratégia de Saúde da Família de Blumenau. Arq Catarin Med [Internet]. 2013 jan-mar [cited 2019 Oct 15];42(1):76-84. Available from: http://www.acm.org.br/revista/pdf/artigos/1216.pdf

12. Brasil. Ministério da Saúde. Vigitel Brasil 2018: Vigilância de fatores de risco e proteção para doenças crônicas por inquérito telefônico: 
estimativas sobre frequência e distribuição sociodemográfica de fatores de risco e proteção para doenças crônicas nas capitais dos 26 estados brasileiros e no Distrito Federal em 2018 [Internet]. Brasília (DF); 2019 [cited 2019 Sep 25]. Available from: https://portalarquivos2.saude.gov.br/images/ pdf/2019/julho/25/vigitel-brasil-2018.pdf

13. Oliveira JLP, Nunes MR. Avaliação da perda da sensibilidade cutânea dos pés de pacientes com diabetes mellitus. Rev Perquirere [Internet]. 2014 jul [cited 2019 Jan 10];1(11):237-46. Available from: https://revistas.unipam.edu.br/index.php/ perquirere/issue/view/103

14. Ravazzani AC, Micali ACP, Lemos D, Santos LDE, Guerra MIM. Risco de úlceras de membros inferiores nos diabéticos de um ambulatório universitário. Rev Med UFPR. 2016;3(2):70-6. DOI: 10.5380/rmu.v3i2.46437

15. Thomazelli FCS, Machado CB, Dolçan KS. Análise do risco de pé diabético em um ambulatório interdisciplinar de diabetes [Internet]. Rev AMRIGS. 2015 jan-mar [cited 2019 Aug 28];59(1):10-4. Available from: https://pesquisa.bvsalud.org/ portal/resource/pt/biblio-836790

16. Rossaneis MA, Haddad MCFL, Mathias TAF, Marcon SS. Diferenças entre mulheres e homens diabéticos no autocuidado com os pés e estilo de vida. Rev Latino-Am Enfermagem. 2016;24:e2761. DOI:10.1590/1518-8345.1203.2761

17. Carvalho RDP, CarvalhoCDP, Martins DA. Aplicação dos cuidados com os pés entre portadores de diabetes mellitus. Cogitare Enferm [Internet]. 2010 jan/mar [cited 2019 Sep 13];15(1):106-9. Available from: https://revistas.ufpr.br/cogitare/article/view/ 17180/11315

18. Martin VT, Rodrigues CDS, Cesarino CB. Conhecimento do paciente com diabetes mellitus sobre o cuidado com os pés. Rev enferm UERJ. 2011 out/dez [cited 2019 Sep 13];19(4):621-5. Available from: http://www.facenf.uerj.br/v19n4/ v19n4a20.pdf
19. Guimarães JPC. Classificação de risco para pé diabético em pessoas idosas com diabetes mellitus tipo 2 [dissertação]. Belo Horizonte (MG): Universidade Federal de Minas Gerais; 2011.

20. Boell JEW, Ribeiro RM, Silva DMGV. Fatores de risco para o desencadeamento do pé diabético. Rev Eletr Enf. 2014 abr/jun;16(2):386-93. DOI: 10.5216/ree.v16i2.20460

21. Bezerra GC, Santos ICRV, Lima JC, Souza MAO. Avaliação do risco para desenvolver pé diabético na atenção básica. Rev Estima [Internet]. 2015 [cited 2019 Oct 3];13(3). Available from: https:// www.revistaestima.com.br/index.php/estima/ article/view/108

22. Paula DB, Martins DA, Lara MO, Stuchi RAG, Lima AMJ, Azevedo DSS. Avaliação dos pés em indivíduos portadores de diabetes atendidos em uma unidade de atenção primária. Rev Enferm UFPE online. 2016 dez;10(Supl 6):S4751-56. DOI: 10.5205/reuol.8200-71830-3-SM.1006sup201601

23. Xia N, Morteza A, Yang F, Cao H, Wang A. Review of the role of cigarette smoking in diabetic foot. J Diabetes Investig. 2019 Mar;10(2):202-12. DOI: $10.1111 /$ jdi.12952

24. Liu M, Zhang W, Yan Z, Yuan X. Smoking increases the risk of diabetic foot amputation: A metaanalysis. Exp Ther Med. 2018 Feb;15(2):1680-5. DOI: $10.3892 /$ etm. 2017.5538

25. Clemens RK, Anemma W, Baumann F, Roth-Zetzsche, Seifert B, von Eckardstein A, et al. Cardiac biomarkers but not measures of vascular atherosclerosis predict mortality in patients with peripheral artery disease. Clin Chim Acta. 2019 Aug;495:215-20. DOI: 10.1016/j.cca.2019. 04.061

Recebido: 30 de outubro de 2019

Aprovado: 16 de janeiro de 2020

Publicado: 15 de abril de 2020

A Revista Baiana de Enfermagem utiliza a Licença Creative Commons - Atribuição-NãoComercial 4.0 Internacional.

https://creativecommons.org/licenses/by-nc/4.0/

Este artigo é de acesso aberto distribuído sob os termos da Licença Creative Commons (CC BY-NC).

Esta licença permite que outros remixem, adaptem e criem a partir do seu trabalho para fins não comerciais. Embora os novos trabalhos tenham de lhe atribuir o devido crédito e não possam ser usados para fins comerciais, os usuários não têm de licenciar esses trabalhos derivados sob os mesmos termos. 